\title{
REVIEW
}

Open Access

\section{Exploring different approaches to improve the success of drug discovery and development projects: a review}

\author{
Geoffrey Kabue Kiriiri ${ }^{{ }^{*}}$ DD, Peter Mbugua Njogu ${ }^{2}$ and Alex Njoroge Mwangi ${ }^{1}$
}

\begin{abstract}
Background: There has been a significant increase in the cost and timeline of delivering new drugs for clinical use over the last three decades. Despite the increased investments in research infrastructure by pharmaceutical companies and technological advances in the scientific tools available, efforts to increase the number of molecules coming through the drug development pipeline have largely been unfruitful.

Main body: A non-systematic review of the current literature was undertaken to enumerate the various strategies employed to improve the success rates in the pharmaceutical research and development. The review covers the exploitation of genomics and proteomics, complementarity of target-based and phenotypic efficacy screening platforms, drug repurposing and repositioning, collaborative research, focusing on underserved therapeutic fields, outsourcing strategy, and pharmaceutical modeling and artificial intelligence. Examples of successful drug discoveries achieved through application of these strategies are highlighted and discussed herein.
\end{abstract}

Conclusions: Genomics and proteomics have uncovered a wide array of potential drug targets and are facilitative of enhanced scrupulous target identification and validation thus reducing efficacy-related drug attrition. When used complementarily, phenotypic and target-based screening platforms would likely allow serendipitous drug discovery while increasing rationality in drug design. Drug repurposing and repositioning reduces financial risks in drug development accompanied by cost and time savings, while prolonging patent exclusivity hence increased returns on investment to the innovator company. Equally important, collaborative research is facilitative of cross-fertilization and refinement of ideas, while sharing resources and expertise, hence reducing overhead costs in the early stages of drug discovery. Underserved therapeutic fields are niche drug discovery areas that may be used to experiment and launch novel drug targets, while exploiting incentivized benefits afforded by drug regulatory authorities. Outsourcing allows the pharma industries to focus on their core competencies while deriving greater efficiency of specialist contract research organizations. The existing and emerging pharmaceutical modeling and artificial intelligence softwares and tools allow for in silico computation enabling more efficient computer-aided drug design. Careful selection and application of these strategies, singly or in combination, may potentially harness pharmaceutical research and innovation.

Keywords: Genomics, Proteomics, Phenotypic, Target-based screening, Repositioning, Repurposing, Modeling, Artificial intelligence

\footnotetext{
* Correspondence: kabuekiriiri@gmail.com

1 Department of Pharmaceutics and Pharmacy Practice, School of Pharmacy,

University of Nairobi, P.O. Box 19676-00202, Nairobi, Kenya

Full list of author information is available at the end of the article
}

\section{Springer Open}

(c) The Author(s). 2020 Open Access This article is licensed under a Creative Commons Attribution 4.0 International License, which permits use, sharing, adaptation, distribution and reproduction in any medium or format, as long as you give appropriate credit to the original author(s) and the source, provide a link to the Creative Commons licence, and indicate if changes were made. The images or other third party material in this article are included in the article's Creative Commons licence, unless indicated otherwise in a credit line to the material. If material is not included in the article's Creative Commons licence and your intended use is not permitted by statutory regulation or exceeds the permitted use, you will need to obtain permission directly from the copyright holder. To view a copy of this licence, visit http://creativecommons.org/licenses/by/4.0/. 


\section{Background}

Humans have been in a perpetual tug-of-war with diseases since the ancient days. Efforts to contain plagues have been recorded in historical artifacts over the course of our existence. While many remedies had been discovered in the early centuries, it cannot be gainsaid that the twentieth century was a pharmaceutical golden era that brought the bulk of the current repertoire of drugs at our disposal [1]. The accelerated speed at which drugs were discovered can be attributed in part by a significant leap in the scientific disciplines of biology and organic chemistry. The former facilitated a thorough understanding of the pathophysiological basis of the diseases hence enabled scientists to accurately detail the underlying biochemical derangements leading to the observed disease phenotype. Organic chemistry, on the other hand, was instrumental in the synthetic and/or semisynthetic derivation of novel drug molecules to address the existing and emerging unmet medical needs [2]. Serendipitous drug discovery also played a critical role in drug discoveries as exemplified by the manner that penicillins were discovered by the English bacteriologist Alexander Fleming in the year 1928. The golden era of drug discoveries continued for five decades following the discovery of penicillin. The resultant effects of drug discoveries were felt across all spheres of the human race most notably the significant improvements in the quality of life and the prolonged longevity enabling humans to live much longer than ever before [3].

The number of new drug molecules coming through the drug discovery and development pipeline started dwindling in the 1980s [4]. A review of the literature reveals that less than one in 10,000 potential drug compounds that begin the drug discovery journey find their way to the clinic [5]. It is hypothesized that we may have exhausted the low hanging fruits and thereby greater efforts are needed to bring new drugs to the market. The entry bar for new molecules entering clinical utilization has been raised by regulatory agencies which demand that significant advantages over existing therapeutic options be evident for the new drugs to be considered for marketing authorization. These attributes include increased efficacy, higher potency, reduced toxicity, ease of administration, and affordability [6]. The overreliance on high technology platforms to identify lead compounds coupled with combinatorial chemistry have been associated with yielding highly lipophilic (greasy) molecules that exhibit poor aqueous solubility resulting in poor pharmacokinetics profiles [7]. These factors have individually and collectively conspired to increase the cost of identifying and developing new drug molecules with the costs currently hovering above US $\$ 2.6$ billion per molecule. The number of pharma companies with such financial clout and willing to take the financial risk has gradually decreased through mergers and acquisitions over the years [8].

While most of the diseases that affect humans have satisfactory therapeutic options available, others have limited or ineffective treatment alternatives and continue exerting a heavy burden on countries and societies. Some of the diseases with huge unmet medical need include neoplastic conditions, diabetes, Alzheimer's disease, immunological disorders, the human immunodeficiency virus-associated acquired immune deficiency syndrome (HIV-AIDs) [9], neglected tropical diseases (NTDs), and rare diseases [10]. Absolute curative therapies for these diseases remain elusive providing a compelling necessity for continued search for new drug molecules.

Figure 1 summarizes the drug discovery and development process. Though a highly lucrative and rewarding enterprise, the process of drug discovery and development is a complicated and arduous scientific journey that begins with identification of a disease or disease area with an unmet medical need. The pharmaceutical or biopharmaceutical firm embarks on the pre-discovery phase which entails elucidation of underlying molecular basis of the disease and development of appropriate animal disease models as well as assay platforms. This is followed by identification of putative targets whose chemical modulation may lead to a therapeutic effect. Upon target identification and validation, the drug discovery team embarks on identifying molecules with the desired pharmacological activity starting with primary hit compounds that are systematically modified to enhance the potency, decrease unwanted effects, and improve desirable physicochemical attributes during the hit-to-lead discovery phase. The end product of the drug discovery process is a candidate drug that is taken through pre-clinical studies and later drug development that transforms the molecule to a clinically useful medicinal product whose efficacy, safety, dosing, and tolerability is established through elaborately designed and executed clinical trials [11].

\section{Strategies for improved success in the drug discovery and development process Key approaches}

Several strategic approaches to enhance efficiency in the drug discovery and development process have been proposed, adopted, and exploited to varied extent in the pharmaceutical research and development (R\&D) projects. They include exploitation of genomics and proteomics, the complementarity of phenotypic and target-based screening platforms, expanding the use of existing drug molecules through repurposing and repositioning, use of collaborative research, exploring under- 


\begin{tabular}{|c|c|}
\hline Prediscovery & $\begin{array}{l}\text { - Exploration of underlying molecular mechanisms of a disease or clinical } \\
\text { syndrome } \\
\text { - Target identification \& validation } \\
\text { - Development of animal disease models } \\
\text { - Assay development }\end{array}$ \\
\hline $\begin{array}{l}\text { Hit-to-lead } \\
\text { discovery }\end{array}$ & $\begin{array}{l}\text {-Identification of primary hit compounds } \\
\text { - Identification \& validation of secondary hit compounds } \\
\text { - Identification \& validation of lead compounds } \\
\text { - Lead optimization } \\
\text { - Selection of development candidate }\end{array}$ \\
\hline $\begin{array}{l}\text { Preclinical } \\
\text { studies }\end{array}$ & $\begin{array}{l}\text { - Toxicity tests in animals in vivo } \\
\text { - Establishment of No adverse effect level (NOAEL) of a development } \\
\text { candidate } \\
\text {-Pharmacokinetics \& pharmacodynamics studies in whole animals } \\
\text { - Preformulation studies }\end{array}$ \\
\hline $\begin{array}{c}\text { Drug } \\
\text { developm }\end{array}$ & $\begin{array}{l}\text { - Clinical trials in humans: Phases I-IV } \\
\text { - Review for marketing authorization }\end{array}$ \\
\hline
\end{tabular}

Fig. 1 Generic outline of the drug discovery and development process

served therapeutic areas, outsourcing approach, and pharmaceutical modeling and artificial intelligence.

\section{Exploitation of genomics and proteomics}

It is an established fact that majority of diseases have a molecular or genetic etiology $[12,13]$. Some conditions including sickle cell disease, cystic fibrosis, muscular dystrophy, and Huntington disease are caused by single gene mutations [14]. Syndromic conditions such as diabetes and cardiovascular diseases have multifactorial causes including multiple gene mutations confounded by environmental and lifestyle factors [12]. In the concept of drug discovery, genes have therefore been classified as disease genes, disease-modifying genes, and druggable genes [15]. Disease genes are those whose mutations cause or predispose a person to the development of a given disease [16]. Disease-modifying genes encode functional proteins whose altered expression is directly linked to the etiology and progression of a given disease. Druggable genes encode proteins that possess recognition domains capable of interacting with drug molecules eliciting a pharmacological response [17].

In the current era of target-based drug discovery, it is imperative that the target is scrupulously identified and validated to establish its essentiality in the disease phenotype. This prevents downstream attrition with available data indicating that a significant proportion (52\%) of drug failure in clinical trials is due to poor efficacy. Figure 2 depicts the various causes of attrition [18, 19]. Classical cases of the drugs imatinib and trastuzumab exemplifies the value of careful target identification and validation in enhancing the success of the drug discovery process [2022]. While the above were new molecules carefully designed with the knowledge of the underlying genetic mutation, existing drugs may find new applications through repositioning from their approved indications based on information obtained through genomics [23]. Genomics can be used to identify and validate druggable

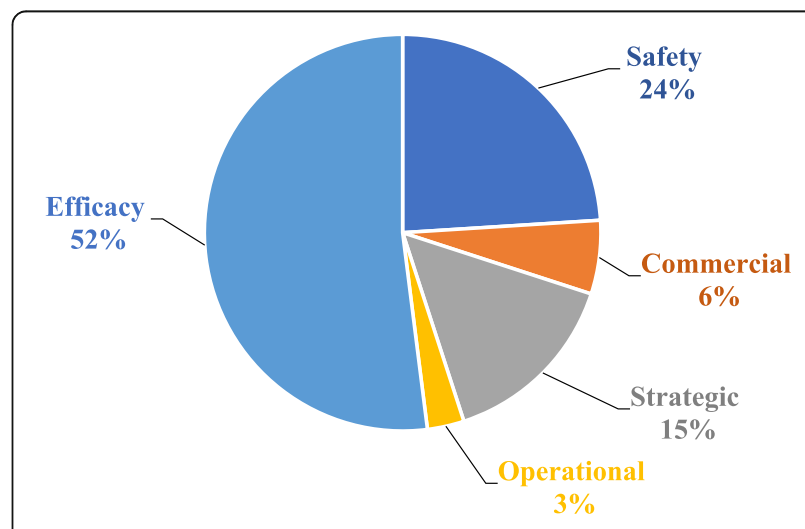

Fig. 2 Causes of attrition in drug discovery and development 
genes thus expanding the number of targets available for exploration in drug discovery $[17,24]$. The use of genomics in target validation has expansively widened through advancement in antisense technology, small interfering RNA (siRNA) that mimic the natural RNA interference (RNAi) and transgenic animal models [25].

Exploitation of genomics is not restricted to target identification and validation. Rather, recent trends in pharma $R \& D$ show that genomics may be employed in the recruitment of study participants for clinical trials with the selection favoring those subjects more likely to benefit from the intervention being trialed. This ensures that the effect of the drug will be evident if the drug is indeed effective against the target disease and absent if ineffective. The outcome so observed would therefore be attributable to the therapeutic intervention and shielded from other confounders. Genomics can also be used as a predictive tool to forecast potential toxicities emanating from a specific molecule [22]. Not surprising, the discipline of pharmacogenomics where drugs are adapted to meet individual profiles is fast gaining traction among researchers and medical practitioners, and has positively impacted the process of drug discovery and development [22].

The human genome was fully described in the year 2002, uncovering a vast treasure trove from which a wide array of novel drug targets could be discovered. Nonetheless, the scientific hype that was associated with the genome project has not been followed with solid benefits as less than 500 of the potential 10,000 targets have been utilized according to the repertoire of drugs registered by the United States Food and Drug Administration (US-FDA) [1, 26]. These targets are protein molecules including DNA, RNA, G protein-coupled receptors (GPCRs), enzymes, and ion channels. The GPCRs constitute the largest proportion of targets for currently registered molecules [27]. It is however expected that the genomic revolution will enhance the drug discovery process significantly given the intensive research currently being done in this field [28].

Proteomics which is a subset of genomics has been widely explored as an avenue of drug discovery [29]. Proteomics entails identification, characterization, and quantification of cellular proteins with the aim of establishing their role in the disease progression and the underlying potential for chemotherapeutic manipulation [25]. Proteomics has been applied widely in drug discovery projects for antineoplastics, neurological, cardiovascular, and rare diseases [30]. Technologies used in proteomics include gel electrophoresis for protein separation and characterization, mass spectrometry (MS) for identification, and yeast hybrid systems to study proteinprotein interactions [31]. These approaches have the potential to identify novel drug targets and their corresponding genes.

\section{Complementarity of phenotypic and target-based screening platforms}

Two distinct screening approaches are routinely employed in the efficacy studies, namely phenotypic (whole-cell) screening and target-based (biochemical) screening. Phenotypic screening evaluates the effects of potential drugs on cultured cell lines (in vitro), isolated tissues/organs (ex-vivo), or in whole animals (in vivo) while target-based screening involves testing the molecules on purified target proteins in vitro [32]. In the first instance, phenotypic screens are primarily aimed at identifying molecules capable of eliciting the desired pharmacological effect without necessarily elucidating the underlying mechanism of action at the molecular level. They are therefore empirically driven as they focus on phenotypic endpoints. Phenotypic drug screening is information-rich, and the therapeutic relevance of the drug is established much earlier in the drug discovery process. The approach is more physiologically relevant as it is conducted in biological systems that simulate the real physiological environment where cognizance that pharmacological effects result from an interplay of many factors is well appreciated [33, 34]. It also provides a huge biological space for serendipitous drug discoveries $[32,35]$. On the contrary, target-based screening is hypothesis-driven, systematic, and rational. Of essence, it requires identification and isolation of a biochemical target whose modulation leads to a desired pharmacological effect. It employs advanced molecular technologies and biological methods that are facilitative of high throughput screening (HTS) platforms [36].

Whereas phenotypic screening predominated in the decades before 1980, it has largely been de-emphasized as advances in molecular biology, and genomics took root and favored the target-based screening [37]. The significant decline in the discovery of first-in-class molecules has in part been attributed to an increasing emphasis on the target-based drug discovery approach [34]. Analysis of data of the drugs registered by the US-FDA reveals that phenotypic drug discovery has yielded more first-in-class molecules than target-based screening [38]. These findings have been challenged by a study that established that 78 of 113 first-in-class molecules registered between years 1999 and 2013 were discovered using target-based screening approaches [39]. The key disadvantages of phenotypic assays include low screening capacity when whole animals are used and the impracticality or difficulty of developing appropriate disease models such as for Alzheimer's disease [40]. Numerous reports have demonstrated the inaccuracy of animal models as tools in predicting therapeutic efficacy in humans [41].

Target-based drug discovery has been the predominant approach of screening putative molecules in the last 
three decades $[33,42]$. This has majorly been due to advances in cloning technologies that allow isolation of pure proteins that are then used to screen a large library of compounds using HTS. The high screening capacity afforded by this approach has cemented target-based platform as the default drug discovery approach as companies seek a competitive edge to deliver novel molecules to the market [36]. Target-based drug discovery begins with understanding the pathophysiological basis of the disease and subsequent identification of the errant biochemical pathway that leads to the disease phenotype. The specific protein that is aberrantly expressed is identified, isolated and its role in the disease phenotype validated by modulation using genomic or pharmacological approaches.

Target-based drug discovery, therefore, elucidates the specific mechanism through which potential drugs produce a pharmacological response. While it lags behind the phenotypic drug discovery approach in yielding firstin-class molecules, target-based drug discovery is unrivalled in producing the best-in-class follower molecules [38]. This is due in part to the rational, hypothesis and systematic approach employed leading to highly selective, potent molecules with better pharmacokinetic and toxicological profiles. Target based-drug discovery has the advantages of being simpler to undertake, enable faster development, and it enables elucidation of the underlying mechanism of action. It also enables the utilization of modern technological advances including computational modeling, molecular biology, combinatorial chemistry, proteomics, and genomics. Conversely, since the approach is based on the modulation of isolated protein targets, the observed effect may have little physiological relevance as there is oversimplification of the physiological environment in which the drug molecules are evaluated [43].

Pharmacological effects derive from complex interactions in intact physiological systems that are best simulated by phenotypic drug discovery and are therefore more predictive of the ultimate therapeutic effect in human disease compared to target-based approaches. It is however imperative that drugs be rationally designed to afford specificity thus improved toxicological profiles, while also providing well-defined mechanisms of action of the pharmacologically active molecules offering a firm foundation upon which drugs with better pharmacokinetics and pharmacodynamics profiles may be developed. Therefore, complementary application of both approaches will invariably lead to increased efficiency in drug discovery with the phenotypic approach delivering first-in-class molecules with proven efficacy early in the discovery process. Target-based drug discovery will build upon these foundations to deliver superior follower molecules employing the knowledge on the molecular interactions of the active molecules with the target. There has been a resurgence of the use of phenotypic drug discovery process in an effort to reverse the decline in discovery of new molecular entities coming through the drug discovery pipeline [34, 44, 45]. Table 1 gives a summary of the merits and demerits of either approach.

\section{Repurposing and repositioning of existing drug molecules}

Drugs that have been developed for a specific therapeutic application may in the course of their clinical use potentially reveal beneficial effects in other therapeutic areas outside the scope of their original indications. These molecules may, therefore, be evaluated for use in the new diseases areas without requiring structural modifications (drug repurposing) [46]. Alternatively, the drugs may require alteration of the primary molecular structure to accentuate a desirable side activity while diminishing the primary effect (drug repositioning) [47]. The two approaches have the potential to resuscitate/ rescue previously abandoned molecules as well as expanding the therapeutic applications of drugs in current use. Examples of successful applications of drug repurposing and repositioning are given in Table 2. They include the drug miltefosine which was developed in the 1980 s as an antitumor agent but abandoned due to dose-limiting gastrointestinal side effects. The drug was refocused as an antileishmanial drug with significant success [49]. Other potential applications for its use as an anti-infective agent have been established with the latest, being its use in the treatment of granulomatous amoebic encephalitis [50].

Sildenafil is another classic example of successful drug repurposing. Although primarily researched for and originally launched into the market for treatment of pulmonary arterial hypertension secondary to patent ductus arteriosus, sildenafil and other phosphodiesterase type 5 inhibitors are best known for their repurposed clinical indication, namely the management of erectile dysfunction [51, 52]. Similarly, drug repositioning was efficiently applied in the R\&D of antidiabetic sulfonylureas from sulfonamide antibiotics where the hypoglycemic effect was enhanced while diminishing the antibacterial effect through systematic structural modifications [53]. The key advantage of drug repurposing and repositioning is the faster development time since the pharmacokinetics and toxicological data as well as other pertinent information regarding the molecules are already available with resultant huge economic savings $[8,46]$. Repurposing remains a viable approach to availing medicines for protozoan diseases and helminthic diseases [54]. Many experimental drugs that were abandoned due to development issues or efficacy shortfalls could be resuscitated through repurposing/repositioning [54]. Approaches to 
Table 1 Advantages and disadvantages of phenotypic and target-based drug discovery approaches

\begin{tabular}{|c|c|}
\hline Advantages & Disadvantages \\
\hline \multicolumn{2}{|l|}{ Phenotypic drug discovery } \\
\hline $\begin{array}{l}\text { Allows establishment of the therapeutic relevance early in the drug } \\
\text { discovery process }\end{array}$ & Difficulties in modeling some diseases \\
\hline Does not require knowledge of the mechanism of action & Policy changes considering animal rights activism \\
\hline Enhances the chances of serendipitous discoveries & $\begin{array}{l}\text { Does not allow utilization of high technology platforms; low screening } \\
\text { capacity }\end{array}$ \\
\hline High chances of discovering first in c class molecules & Complicated analysis due to confounding factors \\
\hline The process closely simulates the normal physiological environment & Rational drug design is not applicable with this model \\
\hline \multicolumn{2}{|l|}{ Target-based drug discovery } \\
\hline Allows elucidation of the mechanism of action & $\begin{array}{l}\text { Observed responses may not be physiologically relevant in the natural } \\
\text { environment }\end{array}$ \\
\hline Very high chances of developing best in class molecules & Requires knowledge of the underlying mechanism of action a priori \\
\hline Allows screening of vast chemical libraries; high screening capacity & Chances of serendipitous drug discovery are minimized \\
\hline Allows utilization of high technology platforms & \\
\hline
\end{tabular}

repurpose or reposition existing drugs include experimental screening and in silico approaches with the latter utilizing data of existing drugs to identify new molecule with the potential clinical application [47].

\section{Collaborative research}

By its nature, the corporate pharmaceutical industry is highly competitive with each company aspiring to dominate the race to launch new blockbuster molecules. It is an established industry fact that early market entrants reap more than those who launch follower molecules. Pioneer companies are able to establish strong brand recognition as well as patient and physician loyalty before competition enter the market [55]. Further, early entrants have sufficient time to perfect their product and set the market price. At any given time, the pharma companies are working to discover and develop molecules addressing similar or very closely related drug targets. Given the astronomical funding channeled into pharmaceutical $R \& D$, these duplicated research efforts collectively end up utilizing resources that could better be invested in the $\mathrm{R} \& \mathrm{D}$ of other disease areas with unmet medical needs. A number of collaborative arrangements have been proposed and utilized for greater success of the pharma R\&D. These include precompetitive research, pharma-academia collaboration, and public-private partnerships (PPP) models [56].

The precompetitive research entails collaboration among pharmaceutical companies, biotechnology companies, and the academic drug discovery units that would otherwise compete but are brought together by a common desire to conduct fundamental research that is

Table 2 Examples of successfully repurposed drugs

\begin{tabular}{|c|c|c|}
\hline Drug & Original indication & Repurposed indication \\
\hline Zidovudine & Anticancer & Antiretroviral \\
\hline Miltefosine & Anticancer & Leishmaniasis \\
\hline Sildenafil & Pulmonary arterial hypertension & Erectile dysfunction \\
\hline Thalidomide & Sedative & Erythema nodosum and multiple myeloma \\
\hline Sirolimus & Immunosuppressant & Lymphoproliferative syndrome \\
\hline Bupropion & Antidepressant & Smoking cessation aid \\
\hline Rituximab & Anticancer & Rheumatoid arthritis \\
\hline Raloxifene & Osteoporosis & Breast cancer \\
\hline Gabapentin & Antiseizure & Post herpetic neuralgia \\
\hline Eflornithine & Cancer & Hirsutism \\
\hline Minoxidil & Antihypertensive & Alopecia \\
\hline Pramipexole & Parkinson's disease & Restless leg syndrome \\
\hline
\end{tabular}


facilitative of subsequent drug discovery and innovation. In essence, precompetitive research establishes scientific viability of pursuing a given therapeutic pathway prior to initiation of full-throttle drug discovery and development campaign. Some of the areas in which precompetitive research may be practiced include target identification and validation, sharing of compound libraries, and biomarker and assay development. There are numerous benefits deriving from precompetitive collaboration including reduced costs of research as companies share their resources and expertise, greater efficiency as companies focus on their core competencies thus furthering their excellence, and crossfertilization of scientific ideas [57]. Precompetitive collaborations are modeled as virtual institutions with scheduled video conferences to monitor and evaluate the progress made. Once the objectives set upon are attained, companies can then venture into separate drug discovery projects [58]. Renown precompetitive collaborations include the Biomarkers Consortium, Innovative Medicine Initiative and TranSMART [59]. TransMART is an inter-organizational collaboration including government agencies, academia, and patient advocacy groups that serves as an open data warehouse arising from clinical trials and basic research [60, 61]. In recognition of the potential gains that could accrue from precompetitive collaborations, the US-FDA developed guidelines for registration of drugs discovered through collaborative strategies in 2011 [62].

There exists a strong justification for pharmaacademia collaboration. While the pharma industry has the financial muscle to fund drug discovery and development programs, the academia boasts of unrivaled proficiency in the conduct of basic research that delivers lead compounds, animal disease models, and putative drug targets [63]. The research capacities of academic institutions have been supported by the availability of tools for translational research, HTS, and chemical libraries. Notable pharma-academia collaborations include AstraZeneca-Columbia University, Pfizer-University of California at San Francisco, Monsanto-University of Washington, and the GlaxoSmithKline (GSK)-Harvard University, among others. The most successful partnerships have been in the area of infectious diseases with drug discoveries for malaria and meningitis A being made [64]. NovoNordisk has successfully employed these partnerships to maintain a competitive edge in the field of diabetes and cardiovascular medicine [65]. Other successful examples include the Scottish Translational Medicine Research Collaboration, the Dundee kinase consortium, structural genetics consortium, Single Nucleotide Polymorphism (SNP) consortium, and the Transcelerate consortium in the USA [66].
The PPP models play an important role in bringing new drugs to patients. The partnerships involve public institutions, pharmaceutical industries, and the academia. These partnerships help improve the productivity in the pharmaceutical industry while also aiding the development of drug discovery capabilities in academia from publicly funded research [67]. The World Health Organization-sponsored Special Programme for Research and Training in Tropical Diseases (WHO/TDR) is one of the most notable PPP globally credited with the discovery of several drugs for tropical diseases. They include chlorproguanil-dapsone combination (Lapdap ${ }^{\circ}$ ) with GSK; injectable artemether with Rhone Poulenc Rorer and injectable $\beta$-arteether with Artecef for malaria; eflornithine with Marion-Merrill Dow for human African trypanosomiasis; miltefosine with Zentaris; and liposomal amphotericin B with NeXstar for visceral leishmaniasis; ivermectin with Merck for onchocerciasis; and praziquantel with Bayer for schistosomiasis $[68,69]$.

\section{Under-served therapeutic fields}

Strategic considerations are vital before a company commits to a drug discovery project. Among the key considerations is the economic viability of a potential drug molecule upon market entry. For sustainable pharma $R \& D$, any drug development candidate must have an acceptable return on investment to ensure the discovery company remains a viable going concern and is able to fund other drugs in the research pipeline. As such majority of the pharmaceutical R\&D efforts are inclined to the therapeutic areas with vast economic potential such as oncology, immunotherapy, endocrinology, neurology, and cardiovascular fields where the probability of recouping the huge capital investment is more certain [41]. Therapeutic areas that offer negligible financial benefits such NTDs and rare diseases do not attract much attention and therefore the opportunities for novel discoveries largely remain unexplored [70]. Rare diseases are genetic disorders that afflict a small patient population and thus offer little economic promise. The NTDs, on the other hand, are vector-borne diseases that afflict billions of people in resource-poor countries. However, these populations have low purchasing power and as such, the pharma companies may not recoup their investments let alone enjoy profitability [71].

The NTDs and rare diseases therapeutic areas present potential avenues of discovering novel drug targets that can then be exploited in other more profitable disease areas where they can be of huge economic value. $\mathrm{Na}$ tional agencies have incentivized pharma $R \& D$ in these areas by providing tax breaks, accelerated reviews, and extended patent exclusivity [72]. Investments in orphan drugs can serve as a solid platform for new molecules providing a safety net for companies, thus reducing the 
impact caused by patent expirations on blockbuster medicines [73]. The rate at which antibiotic resistance is developing outstrips the rate of their development thereby resulting in a decline in the options available for treating infectious diseases. This is also a fertile avenue for pharmaceutical companies to explore [74].

\section{Outsourcing strategies}

The term outsourcing refers to the industrial practice of contracting out services that were previously performed in-house or to access additional capabilities. Outsourcing of certain activities in the drug discovery and development presents an opportunity to enhance the efficiency of the entire process. The outsourcing industry has expanded significantly with the largest growth being registered in China and India where several contract research organizations (CROs) are domiciled supported by cheaper labor, lower land rates, and an increasingly expanding infrastructure [75]. Some of the activities amenable to outsourcing include target identification and validation, development of disease models, lead discovery and optimization, preformulation studies and specific phases or entire clinical trials [76]. This approach allows pharmaceutical companies to focus on their core competencies while delegating specific activities to the more highly specialized CROs.

Since the contracted firms are specialists in their core areas, outsourcing results in faster development and significant economic savings. Research has indicated that clinical trials that are carried out by CROs have higher success rates compared to those executed by pharmaceutical companies [77]. Successful outsourced drug discovery and development projects result in cost reduction, increased operational efficiency, and optimization of resource allocation [78]. Full benefits are only realized when competent partners are selected and the careful implementation of the project followed [79]. Adequate control measures must be instituted to ensure that the contracted organizations follow the established code of ethics while conducting the trials [8].

\section{Pharmaceutical modeling and artificial intelligence}

Modeling entails the use of in silico simulations to predict diverse attributes of a drug molecule including pharmacokinetics and pharmacodynamics profiles [80]. Advances in computing power have enabled development of software that allows simulation of the drugreceptor binding processes, a subset of computer-aided drug design (CADD) also referred to as virtual screening, with tremendous benefits to drug discovery efficiency. First, CADD facilitates generation of focused screens that are then validated in vitro. Second, the CADD is well positioned to guide the lead optimization process thus providing valuable information to the medicinal chemistry team aspiring to enhance the lead molecules receptor affinity or optimize drug metabolism and pharmacokinetics (DMPK) properties including absorption, distribution, metabolism, excretion, and the potential for toxicity (ADMET). Third, the CADD facilitates rational drug design either by "growing" starting molecules one functional group at a time (de novo drug design) on the target site or by piecing together fragments into novel molecules (fragment-based drug design) [81]. Two screening approaches, namely ligandbased virtual screening and target-based virtual screening, have been used in CADD to filter out the compounds that are unlikely to be successful in the development pipeline due to poor physicochemical properties and/or intolerable toxicological profile while identifying those likely to have the activity of interest.

In ligand-based virtual screening, structural features of known compounds are used to construct computer models that are used to predict the properties of other compounds not included in the training data set. The data sets are then used to generate quantitativestructure activity relationship (QSAR) models correlating structural features and the physicochemical properties of a homologous series to the observed biological activity. The chemical structure of known compounds is reduced to a set of molecular descriptors that are used to generate a mathematical model that is used to predict the properties of the test compounds. Molecular descriptors with the highest activity are chosen for the model [82]. Target-based virtual screening entails computer models that test the docking properties of test compounds against the three-dimensional structure of the target (Xray crystal structure or homology model) [83-85]. Each of the test compounds is optimally positioned on the binding site and assigned a score based on the binding affinity. Top scoring compounds are synthesized and tested in vitro [86]. Application of these models can enhance the efficiency of drug discovery projects by providing focused screens that can have better chances of succeeding downstream. Problematic molecules are also identified earlier in the drug discovery process thus avoiding expensive late-stage failures. Integration of ligand-based and target-based virtual screening yields better results [32, 87].

Modeling and simulation have also been employed in various areas of the clinical drug development process [88]. The modeling process is founded on mathematical polynomials generated from empirical data for real-life patients. Key areas that may be modeled include biosimulation to inform planning, implementation, and evaluation of clinical trial designs with the goal of optimizing the efficiency, quality, and cost effectiveness of the trials. Pharmacodynamic and pharmacokinetic 
models are used to predict optimal dosage levels in the various phases of clinical trials as well as in special populations including pediatrics, geriatrics, pregnant women, and others with constrained physiological conditions that will impact on drug disposition. The application of modeling improves the effectiveness of clinical trials with enormous cost and time saving [89, 90]. Successful application of computer-based drug design is exemplified by several drugs in clinical use including nelfinavir, imatinib, zanamivir, saquinavir, and norfloxacin [91].

Artificial intelligence (AI) is increasingly being applied in the drug design and development. This has been possible due to the availability of large chemical and biological databases that are prerequisites for development of accurate predictive models. Scientists contend that AI has the capacity to revolutionize the drug discovery process enabling the screening of billions of potential molecules for hit identification, prioritization of proposed alternatives, and validation of biological targets. It can further guide lead optimization and inform the design and implementation of clinical trials in the latter stages of drug development. Consequently, strategic implementation of AI could enormously supplement the R\&D efforts to avail novel, effective, and safe drugs to alleviate human suffering due to unmet clinical needs [92]. Generative deep learning networks can propose completely new molecules that exhibit the desired physical and biological properties which can be instrumental in the discovery of drugs for complex disease conditions. They may also be in the optimization of existing molecules. AI is also applied in the multi-objective optimization of lead molecules through the application of machine learning allowing identification of compounds that exhibit a healthy balance of the requisite set of physicochemical, biological, and pharmacokinetic characteristics [93]. Examples of software used in pharmaceutical modeling and AI-guided drug discovery are listed in Table 3.

\section{Conclusion}

The ever-increasing costs of drug discovery projects have not translated into increased efficiency in delivering new medicines. On the contrary, fewer drugs are transiting through the drug development pipeline than ever before. The observed productivity decline is majorly attributable to the overreliance of the industry on high technology platforms, stringent drug registration and approval requirements for new medicines, and the exhaustion of the obvious and easy-to-reach drug targets necessitating exploration of more complex biological systems.

Scientific advancements allow the application of advanced molecular techniques that include genomics and lately proteomics in identification and validation of drug targets. Carefully executed target identification and validation will reduce the attrition rates attributable to poor efficacy that currently accounts for more than $50 \%$ of drug failures. The complementarity of phenotypic and targetbased drug discovery approaches would enable discovery of first-in-class molecules while also delivering safer, more efficacious and potent best-in-class follower molecules.

Collaborative strategies, such as precompetitive research and public-private partnerships, have positively impacted efficiency in drug discovery. Expansion of research activities into the underserved therapeutic areas covering rare and neglected diseases would offer a safeguard for companies whose blockbuster drugs are teetering on the patent cliff. Advances in computing technologies will also facilitate selection of focused screens with better success rates downstream. Pharmaceutical modeling and AI are expected to continue contributing significantly to improved efficiency in drug discovery and development in the years to come. Carefully executed outsourcing strategies allow companies to focus on their core competencies while delegating other development activities to expertise offered by the CROs, a strategy that accelerates the discovery process while reducing overhead costs.

Table 3 Examples of pharmaceutical modeling and artificial intelligence-guided drug discovery tools

\begin{tabular}{|c|c|c|}
\hline Tool & Application in drug discovery & Link \\
\hline Alphafold & Target modeling & $\begin{array}{l}\text { https://deepmind.com/blog/article/AlphaFold-Using-Al-for-scientific- } \\
\text { discovery }\end{array}$ \\
\hline AutoDock & Protein ligand-binding modeling & http://autodock.scripps.edu/ \\
\hline DeepTox & Predictive toxicology & https://deeptox.co \\
\hline $\begin{array}{l}\text { Discovery } \\
\text { Studio }\end{array}$ & $\begin{array}{l}\text { Pharmacophore modeling, target identification, lead } \\
\text { optimization }\end{array}$ & $\begin{array}{l}\text { https://www.3dsbiovia.com/products/collaborative-science/biovia- } \\
\text { discovery-studio/ }\end{array}$ \\
\hline Glide & Combinatorial chemistry and docking studies & https://www.schrodinger.com/glide \\
\hline PPB2 & Predictive pharmacology & http://ppb2.gdb.tools/ \\
\hline PotentialNet & Protein-ligand binding and molecular properties modeling & https://pubs.acs.org/doi/full/10.1021/acscentsci.8b00507 \\
\hline Python & Pharmaceutical modeling & https://www.python.org/ \\
\hline
\end{tabular}




\section{Abbreviations}

ADMET: Absorption, distribution, metabolism, elimination, and toxicity; Al: Artificial intelligence; AIDS: Acquired immune deficiency syndrome; CADD: Computer-aided drug design; CRO: Contract Research Organization; DMPK: Drug metabolism and pharmacokinetics; DNA: Deoxyribonucleic acid; US-FDA: United States Food and Drug Administration; GCPRs: G proteincoupled receptors; GSK: GlaxoSmithKline; HTS: High throughput screening; MS: Mass spectrometry; PPP: Public-private partnerships; QSAR: Quantitativestructure activity relationship; R\&D: Research and development; RNA: Ribonucleic acid; RNAi: RNA interference; siRNA: Small interfering RNA; TDR: Special Programme for Research and Training in Tropical Diseases; WHO: World Health Organization

\section{Acknowledgements}

The authors are grateful to the University of Nairobi for providing infrastructural support by way of online resources required for this review.

\section{Authors' contributions}

GK conceived and wrote the first draft; PN and AN reviewed all drafts and gave additional input to improve the scientific rigor required. The authors read and approved the final draft for publication.

\section{Funding}

There was no funding for this manuscript.

\section{Availability of data and materials}

Data and materials are available upon request.

\section{Ethics approval and consent to participate}

Not applicable.

\section{Consent for publication}

Not applicable.

\section{Competing interests}

There are no competing interests to declare for all authors.

\section{Author details}

'Department of Pharmaceutics and Pharmacy Practice, School of Pharmacy, University of Nairobi, P.O. Box 19676-00202, Nairobi, Kenya. ${ }^{2}$ Department of Pharmaceutical Chemistry, School of Pharmacy, University of Nairobi, P.O. Box 19676-00202, Nairobi, Kenya.

Received: 14 November 2019 Accepted: 10 June 2020

Published online: 23 June 2020

\section{References}

1. Drews J (2000) Drug discovery: a historical perspective. Science 287:19601964 https://doi.org/10.1126/science.287.5460.1960

2. Rotella DP (2016) The critical role of organic chemistry in drug discovery. ACS Chem Neurosci 7:1315-1316 https://doi.org/10.1021/acschemneuro. $6 \mathrm{~b} 00280$

3. Gaynes R (2017) The discovery of penicillin — new insights after more than 75 years of clinical use. Emerg Infect Dis 23:849-853 https://doi.org/10.3201/ eid2305.161556

4. Pammolli F, Magazzini L, Riccaboni M (2011) The productivity crisis in pharmaceutical R\&D. Nat Rev Drug Discov 10:428-438 https://doi.org/10. $1038 /$ nrd3405

5. Petrova E et al (2014) Innovation in the pharmaceutical industry: the process of drug discovery and development. Springer, New York, pp 19-8

6. Naci H, Carter AW, Mossialos E (2015) Why the drug development pipeline is not delivering better medicines. BMJ:h5542 https://doi.org/10.1136/bmj. h5542

7. Lipinski CA (2000) Drug-like properties and the causes of poor solubility and poor permeability. J Pharmacol Toxicol Methods 44:235-249. https://doi.org/ 10.1016/S1056-8719(00)00107-6

8. Paul SM, Mytelka DS, Dunwiddie CT et al (2010) How to improve R\&D productivity: the pharmaceutical industry's grand challenge. Nat Rev Drug Discov 9:203-214 https://doi.org/10.1038/nrd3078
9. Cummings JL, Morstorf T, Zhong K (2014) Alzheimer's disease drugdevelopment pipeline: few candidates, frequent failures. Alzheimers Res Ther 6:37 https://doi.org/10.1186/alzrt269

10. Ridley RG (2002) Medical need, scientific opportunity and the drive for antimalarial drugs. Nature 415:686-693 https://doi.org/10.1038/415686a

11. Hughes J, Rees S, Kalindjian S, Philpott K (2011) Principles of early drug discovery: principles of early drug discovery. Br J Pharmacol 162:1239-1249 https://doi.org/10.1111/j.1476-5381.2010.01127.x

12. Wallis G, BASC (1999) The genetic basis of human disease. The Biochemical Society, London

13. King R, Rotter J, Motulksy A (2002) The genetic basis of common diseases, 2nd ed

14. WHO | Genes and human disease. In: WHO. https://www.who.int/genomics/ public/geneticdiseases/en/index2.html. Accessed 31 Jan 2019

15. Hopkins AL, Groom CR (2002) The druggable genome. Nat Rev Drug Discov 1:727-730 https://doi.org/10.1038/nrd892

16. Dixon SJ, Stockwell BR (2009) Identifying druggable disease-modifying gene products. Curr Opin Chem Biol 13:549-555 https://doi.org/10.1016/j.cbpa. 2009.08.003

17. Chris Finan (2017) The druggable genome and support for target identification and validation in drug development. Sci Transl Med 9: eaag1166. https://doi.org/10.1126/scitranslmed.aag1166

18. Harrison RK (2016) Phase II and phase III failures: 2013-2015. Nat Rev Drug Discov 15:817-818 https://doi.org/10.1038/nrd.2016.184

19. Hopkins AL (2008) Network pharmacology: the next paradigm in drug discovery. Nat Chem Biol 4:682-690 https://doi.org/10.1038/nchembio.118

20. Capdeville R, Buchdunger E, Zimmermann J, Matter A (2002) Glivec (STI571, imatinib), a rationally developed, targeted anticancer drug. Nat Rev Drug Discov 1:493-502 https://doi.org/10.1038/nrd839

21. Cobleigh MA et al (1999) Multinational study of the efficacy and safety of humanized anti-HER2 monoclonal antibody in women who have HER2overexpressing metastatic breast cancer that has progressed after chemotherapy for metastatic disease. J Clin Oncol 17:2639-2639 https://doi. org/10.1200/JCO.1999.17.9.2639

22. Garman KS, Nevins JR, Potti A (2007) Genomic strategies for personalized cancer therapy. Hum Mol Genet 16:R226-R232 https://doi.org/10.1093/hmg/ ddm 184

23. Lussier YA, Chen JL (2011) The emergence of genome-based drug repositioning. Sci Transl Med 3:96ps35-96ps35. https://doi.org/10.1126/ scitranslmed.3001512

24. Plenge RM, Scolnick EM, Altshuler D (2013) Validating therapeutic targets through human genetics. Nat Rev Drug Discov 12:581-594 https://doi.org/ $10.1038 /$ nrd4051

25. Lindsay MA (2003) Target discovery. Nat Rev Drug Discov 2:831-838 https:// doi.org/10.1038/nrd1202

26. Penrod NM, Cowper-Sal-lari R, Moore JH (2011) Systems genetics for drug target discovery. Trends Pharmacol Sci 32:623-630 https://doi.org/10.1016/j. tips.2011.07.002

27. Bleicher KH, Böhm H-J, Müller K, Alanine Al (2003) Hit and lead generation: beyond high-throughput screening: a guide to drug discovery. Nat Rev Drug Discov 2:369-378 https://doi.org/10.1038/nrd1086

28. Dimitri Semizarov, Eric Blomme (2008) Genomics in drug discovery and development | Wiley. In: Wiley.com. https://www.wiley.com/en-us/ Genomics+in+Drug+Discovery+and+Development-p-9780470096048. Accessed 19 Mar 2020

29. Chanda SK, Caldwell JS (2003) Fulfilling the promise: drug discovery in the post-genomic era. Drug Discov Today 8:168-174. https://doi.org/10.1016/ S1359-6446(02)02595-3

30. Jain KK (2002) Proteomics-based anticancer drug discovery and development. Technol Cancer Res Treat 1:231-236 https://doi.org/10.1177/ 153303460200100403

31. Jain KK (2004) Applications of proteomics technologies for drug discovery. In: Hondermarck H (ed) Proteomics: Biomedical and Pharmaceutical Applications. Kluwer Academic Publishers, Dordrecht, pp 201-227

32. Moffat JG, Vincent $F$, Lee JA et al (2017) Opportunities and challenges in phenotypic drug discovery: an industry perspective. Nat Rev Drug Discov 16:531-543 https://doi.org/10.1038/nrd.2017.111

33. Wagner BK (2016) The resurgence of phenotypic screening in drug discovery and development. Expert Opin Drug Discovery 11:121-125 https://doi.org/10.1517/17460441.2016.1122589 
34. Zheng W, Thorne N, McKew JC (2013) Phenotypic screens as a renewed approach for drug discovery. Drug Discov Today 18:1067-1073 https://doi org/10.1016/j.drudis.2013.07.001

35. Ban TA (2006) The role of serendipity in drug discovery. Clin Res 8:10

36. Samsdodd F (2005) Target-based drug discovery: is something wrong? Drug Discov Today 10:139-147. https://doi.org/10.1016/S1359-6446(04)03316-1

37. Kotz J (2012) Phenotypic screening, take two. Sci-Bus Exch 5:380-380 https://doi.org/10.1038/scibx.2012.380

38. Swinney DC (2013) Phenotypic vs. target-based drug discovery for first-inclass medicines. Clin Pharmacol Ther 93:299-301 https://doi.org/10.1038/ clpt.2012.236

39. Eder J, Sedrani R, Wiesmann C (2014) The discovery of first-in-class drugs: origins and evolution. Nat Rev Drug Discov 13:577-587 https://doi.org/10. $1038 /$ nrd4336

40. Khachaturian ZS (2002) Models and modeling systems in Alzheimer disease drug discovery. Alzheimer Dis Assoc Disord 16

41. Norris, Diana Pankevich, Miriam Davis, Bruce Altevogt (2014) Improving and accelerating therapeutic development for nervous system disorders: workshop summary. National Academies Press (US) Washington (DC)

42. Swinney DC, Anthony J (2011) How were new medicines discovered? Nat Rev Drug Discov 10:507-519 https://doi.org/10.1038/nrd3480

43. Croston GE (2017) The utility of target-based discovery. Expert Opin Drug Discovery 12:427-429 https://doi.org/10.1080/17460441.2017.1308351

44. Comley J (2015) Phenotypic drug discovery: striving towards the highest level of biological relevance. https://www.ddw-online.com/. Accessed 28 Jan 2019

45. Sams-Dodd F (2006) Drug discovery: selecting the optimal approach. Drug Discov Today 11:465-472 https://doi.org/10.1016/j.drudis.2006.03.015

46. Pushpakom S, lorio F, Eyers PA et al (2018) Drug repurposing: progress, challenges and recommendations. Nat Rev Drug Discov 18:41-58 https:// doi.org/10.1038/nrd.2018.168

47. Cha Y, Erez T, Reynolds IJ et al (2018) Drug repurposing from the perspective of pharmaceutical companies: drug repurposing in pharmaceutical companies. Br J Pharmacol 175:168-180 https://doi.org/10 1111/bph.13798

48. Ashburn TT, Thor KB (2004) Drug repositioning: identifying and developing new uses for existing drugs. Nat Rev Drug Discov 3:673-683 https://doi.org/ 10.1038/nrd1468

49. Charlton RL, Rossi-Bergmann B, Denny PW, Steel PG (2018) Repurposing as a strategy for the discovery of new anti-leishmanials: the-state-of-the-art. Parasitology 145:219-236 https://doi.org/10.1017/S0031182017000993

50. Sindermann H, Engel J (2006) Development of miltefosine as an oral treatment for leishmaniasis. Trans R Soc Trop Med Hyg 100:S17-S20 https:// doi.org/10.1016/j.trstmh.2006.02.010

51. Board on Health Sciences Policy (2014) Drug repurposing and repositioning: workshop summary. National Academies Press (US)

52. White JR (2014) A brief history of the development of diabetes medications. Diabetes Spectr 27:82-86 https://doi.org/10.2337/diaspect.27.2.82

53. Sola D, Rossi L, Schianca GPC et al (2015) State of the art paper sulfonylureas and their use in clinical practice. Arch Med Sci 4:840-848 https://doi.org/10.5114/aoms.2015.53304

54. Andrews KT, Fisher G, Skinner-Adams TS (2014) Drug repurposing and human parasitic protozoan diseases. Int J Parasitol Drugs Drug Resist 4:95111 https://doi.org/10.1016/j.ijpddr.2014.02.002

55. Cha M, Yu F (2018) Pharma's first-to-market advantage | McKinsey. https:// www.mckinsey.com/industries/pharmaceuticals-and-medical-products/ourinsights/pharmas-first-to-market-advantage. Accessed 29 Jan 2019

56. Munos BH, Chin WW (2011) How to revive breakthrough innovation in the pharmaceutical industry. Sci Transl Med 3:89cm16-89cm16. https://doi.org/ 10.1126/scitranslmed.3002273

57. Yildirim O, Gottwald M, Schüler P, Michel MC (2016) Opportunities and challenges for drug development: public-private partnerships, adaptive designs and big data. Front Pharmacol 7. https://doi.org/10.3389/fphar.2016.00461

58. Medicine (US) (2010) Types of pre-competitive collaborations. National Academies Press (US)

59. Gastfriend E, Lee B (2015) Pre-competitive collaboration in pharma. 19

60. Athey BD, Braxenthaler M, Haas M, Guo Y. tranSMART: An Open Source and Community-Driven Informatics and Data Sharing Platform for Clinical and Translational Research. https://www.ncbi.n/m.nih.gov/pmc/articles/PMC3 814495/\#_ffn_sectitle.
61. Schumacher A, et al (2014) A collaborative approach to develop a multiomics data analytics platform for translational research. https://reader. elsevier.com/reader/sd/pii/S2212066114000350?token=086E8A7B05341EE1 C0A558241BF007678C6B310508BD3F97DFD1DEC923B28DE719E0E0DB2B82 F6936FC9E544E850DE7F.

62. Fridlyand J, Simon RM, Walrath JC et al (2013) Considerations for the successful co-development of targeted cancer therapies and companion diagnostics. Nat Rev Drug Discov 12:743-755 https://doi.org/10.1038/ nrd4101

63. Palmer M, Chaguturu R (2017) Academia-pharma partnerships for novel drug discovery: essential or nice to have? Expert Opin Drug Discovery 12: 537-540 https://doi.org/10.1080/17460441.2017.1318124

64. Cui L, Su X (2009) Discovery, mechanisms of action and combination therapy of artemisinin. Expert Rev Anti-Infect Ther 7:999-1013 https://doi. org/10.1586/eri.09.68

65. Stilz HU, Bregenholt S (2018) Successful pharmaceutical innovation: In: Frølund L, Riedel MF (eds) Strategic Industry-University Partnerships. Elsevier, pp 39-57

66. Gill D (2014) Re-inventing clinical trials through TransCelerate. Nat Rev Drug Discov 13:787-788 https://doi.org/10.1038/nrd4437

67. Tralau-Stewart CJ, Wyatt CA, Kleyn DE, Ayad A (2009) Drug discovery: new models for industry-academic partnerships. Drug Discov Today 14:95-101 https://doi.org/10.1016/j.drudis.2008.10.003

68. Nwaka S, Ridley RG (2003) Virtual drug discovery and development for neglected diseases through public-private partnerships. Nat Rev Drug Discov 2:919-928 https://doi.org/10.1038/nrd1230

69. Balaña-Fouce R, Pérez Pertejo MY, Domínguez-Asenjo B et al (2019) Walking a tightrope: drug discovery in visceral leishmaniasis. Drug Discov Today 24: 1209-1216 https://doi.org/10.1016/j.drudis.2019.03.007

70. Hong-Bo Weng et al (2018) Innovation in neglected tropical disease drug discovery and development. 7:

71. Hunter J (2011) Challenges for pharmaceutical industry: new partnerships for sustainable human health. Philos Trans R Soc A Math Phys Eng Sci 369: 1817-1825 https://doi.org/10.1098/rsta.2010.0377

72. Volmar C-H, Wahlestedt C, Brothers SP (2017) Orphan diseases: state of the drug discovery art. Wien Med Wochenschr 167:197-204 https://doi.org/10. 1007/s10354-015-0423-0

73. Sharma A, Jacob A, Tandon M, Kumar D (2010) Orphan drug: development trends and strategies. J Pharm Bioallied Sci 2:290-299 https://doi.org/10. 4103/0975-7406.72128

74. Projan SJ (2003) Why is big pharma getting out of antibacterial drug discovery? Curr Opin Microbiol 6:427-430 https://doi.org/10.1016/j.mib.2003. 08.003

75. Subramaniam S, Dugar S (2012) Outsourcing drug discovery to India and China: from surviving to thriving. Drug Discov Today 17:1055-1058 https:// doi.org/10.1016/j.drudis.2012.04.005

76. Piachaud BS (2002) Outsourcing in the pharmaceutical manufacturing process: an examination of the CRO experience. Technovation 22:81-90. https://doi.org/10.1016/S0166-4972(01)00081-5

77. Kaitin KI (2010) Deconstructing the drug development process: the new face of innovation. Clin Pharmacol Ther 87:356-361 https://doi.org/10.1038/ clpt.2009.293

78. Shuchman M (2007) Commercializing clinical trials — risks and benefits of the CRO boom. N Engl J Med 357:1365-1368 https://doi.org/10.1056/ NEJMp078176

79. Klopack TG (2000) Balancing the risks and the benefits. Drug Discov Today 5:157-160. https://doi.org/10.1016/S1359-6446(00)01469-0

80. Klebe G (2006) Virtual ligand screening: strategies, perspectives and limitations. Drug Discov Today 11:580-594 https://doi.org/10.1016/j.drudis. 2006.05.012

81. Song CM, Lim SJ, Tong JC (2009) Recent advances in computer-aided drug design. Brief Bioinform 10:579-591 https://doi.org/10.1093/bib/bbp023

82. Roy K, Kar S, Das RN (2015) Understanding the basics of QSAR for applications in pharmaceutical sciences and risk assessment, 1st ed. Academic Press

83. Agah S, Faham S, Vaidehi N, Klein-Seetharaman J (2012) Membrane protein structure and dynamics: methods and protocols, 1st ed. Humana Press

84. Lyne PD (2002) Structure-based virtual screening: an overview. Drug Discov Today 7:1047-1055. https://doi.org/10.1016/S1359-6446(02)02483-2

85. Cole JC, Korb O, Olsson TSG, Liebeschuetz J (2011) The basis for targetbased virtual screening: protein structures. In: Virtual Screening. pp 87-114 
86. Lindahl ER, Kukol A (2008) Molecular modeling of proteins, 1st ed. Humana Press

87. Wiggers HJ, Rocha JR, Cheleski J, Montanari CA (2011) Integration of ligandand target-based virtual screening for the discovery of cruzain inhibitors. Mol Inform 30:565-578 https://doi.org/10.1002/minf.201000146

88. Stockmann C, Barrett J, Roberts J, Sherwin C (2015) Use of modeling and simulation in the design and conduct of pediatric clinical trials and the optimization of individualized dosing regimens. CPT Pharmacometrics Syst Pharmacol 4:630-640 https://doi.org/10.1002/psp4.12038

89. Girard P, Cucherat M, Guez D et al (2004) Clinical trial simulation in drug development. Thérapie 59:297-304 https://doi.org/10.2515/therapie:2004057

90. Office of the Commissioner (2018) About science \& research at FDA - how simulation can transform regulatory pathways. Accessed 1 Feb 2019

91. Prieto-Martínez FD, López-López E, Eurídice Juárez-Mercado K, MedinaFranco JL (2019) Computational drug design methods-current and future perspectives. In: In Silico Drug Design. Elsevier, pp 19-44

92. Chan HCS, Shan H, Dahoun T et al (2019) Advancing drug discovery via artificial intelligence. Trends Pharmacol Sci 40:592-604 https://doi.org/10 1016/j.tips.2019.06.004

93. Schneider P, Walters WP, Plowright AT et al (2019) Rethinking drug design in the artificial intelligence era. Nat Rev Drug Discov https://doi.org/10.1038/ s41573-019-0050-3

\section{Publisher's Note}

Springer Nature remains neutral with regard to jurisdictional claims in published maps and institutional affiliations.

\section{Submit your manuscript to a SpringerOpen ${ }^{\circ}$ journal and benefit from:}

- Convenient online submission

- Rigorous peer review

- Open access: articles freely available online

High visibility within the field

- Retaining the copyright to your article

Submit your next manuscript at $\boldsymbol{\nabla}$ springeropen.com 